\title{
Uma Demonstração de como o Método da Função Zeta para o Potencial Efetivo Elimina as Divergências
}

(Demonstration of how the zeta function method for effective potential removes the divergences)

\author{
José Alexandre Nogueira \\ Departamento de Física, Centro de Ciências Exatas, \\ Universidade Federal do Espirito Santo, \\ 29.060-900 - Vitória-ES - Brasil, \\ E-mail: nogueira@cce.ufes.br
}

Adolfo Maia Jr.

Instituto de Matemática, Universidade Estadual de Campinas, 13.081-970 - Campinas-SP - Brasil

E-mail: maia@ime.unicamp.br

Recebido em 27 de janeiro, 2002. Aceito em 22 de maio, 2002.

\begin{abstract}
O cálculo do potencial efetivo usando o método da função zeta é extremamente vantajoso, porque a função zeta é regular em $s=0$, e nós obtemos imediatamente um resultado finito para o potencial efetivo sem a necessidade de subtração de qualquer pólo ou a adição de contratermos infinitos. O propósito deste artigo é mostrar explicitamente como ocorre o cancelamento das divergências e que o método da função zeta implicitamente usa o mesmo procedimento usado por Bollini-Giambiagi e Salam-Strathdee para obter a parte finita de funções com um pólo simples.
\end{abstract}

The calculation of the minimum of the effective potential using the zeta function method is extremely advantagous, because the zeta function is regular at $s=0$ and we gain immediately a finite result for the effective potential without the necessity of subtratction of any pole or the addition of infinite counter-terms. The purpose of this paper is to explicitly point out how the cancellation of the divergences occurs and that the zeta function method implicitly uses the same procedure used by Bollini-Giambiagi and Salam-Strathdee in order to gain finite part of functions with a simple pole.

\section{Introdução}

Os físicos de altas energias foram os primeiros a perceberem a necessidade de uma teoria quântica de campos relativística e mais tarde físicos de outras especialidades encontraram nela uma poderosa ferramenta. Indubitavelmente a teoria quântica de campos obteve seu primeiro sucesso na eletrodinâmica quântica (QED) e seu maior sucesso no modelo padrão.

A teoria quântica de campos é fundamentalmente de aspecto perturbativo. Portanto, as quantidades com maior interesse físico, as funções de Green, são construídas por meio de séries perturbativas. Contudo, a teoria quântica de campos tem enormes problemas de divergências em todos seus aspectos perturbativos. O tratamento desses infinitos ainda é um dos maiores desafios em teoria quântica de campos. A natureza matemática do problema é clara. Divergências ocorrem nos cálculos perturbativos porque duas distribuições não podem ser multiplicadas no mesmo ponto. Vários métodos têm sidos porpostos para eliminar esse problema. Entretanto, somente tem sido possível eliminar esses infinitos consistentemente e de uma maneira fisicamente aceitável absorvendo-os nos parâmetros nus da teoria (aqueles não físicos).

Uma quantidade de considerável importância física é a densidade de energia do vácuo que está associada com interessantes efeitos físicos, tais como "Lamb Shift" e o Efeito Casimir, os quais ocorrem devido às flutuações do vácuo. Porém, existem diversas variações sobre a determinação da energia do vácuo em uso. Entre elas, o mínimo do potencial efetivo obtido da abor- 
dagem de métodos funcionais da teoria quântica de campos é extensivamente usada. A principal aplicação do potencial efetivo está associada com a quebra espontânea de simetria. O potencial efetivo é obtido de um método não perturbativo como uma série em "loop" $(\hbar)$. No limite clássico, que é a chamada aproximação em árvore, o potencial efetivo torna-se igual ao potencial clássico, portanto ele é o potencial clássico mais correções quânticas. O potencial efetivo também sofre os mesmos problemas de divergências já citados.

O procedimento usual para tratar estas divergências tem sido empregar um método de regularização (dimensional, "cut-off", etc) a fim de isolar as divergências e tornar a teoria finita fazendo-se o uso de um regulador e, depois, usando-se uma prescrição de renormalização, subtração dos pólos ou adição de contratermos, de forma a ajustar os parâmetros da teoria àqueles físicos (observados), eliminar as divergências isoladas e restabelecer a teoria original com a eliminação do regulador. Uma vez que a subtração dos pólos ou a adição de contratermos infinitos, embora bem fundamentada em espaços planos ("flat"), é dúbia em espaços curvos. O cálculo do mínimo do potencial efetivo usando-se o método da função zeta é vantajoso, pois a função zeta é regular em $s=0$ e nós obtemos um resultado finito para o potencial efetivo sem a aparente necessidade de subtração de qualquer pólo ou a adição de contratermos infinitos. Contudo, é óbvio que implicitamente no método da função zeta deve existir o cancelamento das divergências.

O trabalho está organizado da seguinte forma: na seção 2 nós explicitamente mostramos o cancelamento das divergências no método da função zeta; na seção 3 nós mostramos que o método da função zeta é equivalente ao bem conhecido procedimento para obter a parte finita de funções com um pólo simples (neste caso, aquele relacionado á energia de ponto zero) usado por Bollini-Giambigi e Salam-Strathee, isto porque o método da função zeta é um procedimento de regularização analítica; na seção 4 nós fazemos nossas conclusões.

\section{O cancelamento das di- vergências}

$\mathrm{Na}$ abordagem de métodos funcionais da teoria quântica de campos, a densidade de energia do vácuo pode ser determinada pelo cálculo do mínimo do potencial efetivo [1-7]. A densidade de energia desta forma determinada é uma expansão em loop (ou equivalentemente em potências de $\hbar$ ), isto é, sua parte clássica mais correções quânticas.

Seja $\phi(x)$ um campo real escalar em um espaçotempo de Minkowski, sujeito ao potencial $V(\phi)$. O mínimo do potencial efetivo para primeira ordem na expansão em "loop" é dado por

$$
V_{e f}(\bar{\phi})=V_{c l}(\bar{\phi})+\frac{1}{2} \frac{\hbar}{\Omega} \ln \operatorname{det}\left[\frac{\delta^{2} S[\bar{\phi}]}{\delta \phi(x) \delta \phi(y)}\right]=V_{c l}(\bar{\phi})+V_{e f}^{(1)}(\bar{\phi})
$$

onde $\bar{\phi}=\langle\phi\rangle$ é o valor esperado do vácuo, $S[\phi]$ é a ação clássica, $\Omega=V T$ é o volume do espaço-tempo, e no potencial clássico $V_{c l}(\phi)$ estão incluídos termos de massa e auto-interação.

Fazendo-se a usual continuação analítica para o espaço-tempo euclidiano [2,4], a ação clássica pode ser escrita como

$$
S[\phi]=\int d^{4} x\left[\frac{1}{2} \partial_{\mu} \phi \partial_{\mu} \phi+V_{c l}(\phi)\right]
$$

onde uma convenção de somatório euclidiana para índices repetidos está implícita. Da eq.(2) nós obtemos a matriz $m(x, y)$ da segunda variação da ação $S[\phi]$

$$
m(x, y) \equiv \frac{\delta^{2} S\left[\phi_{c}\right]}{\delta \phi(x) \delta \phi(y)}=\delta^{4}(x-y)\left[-\delta^{\mu \nu} \partial_{\mu} \partial_{\nu}+V_{c l}^{\prime \prime}\left(\phi_{c}\right)\right]
$$


O campo clássico $\phi_{c l}(x)=\langle\phi(x)\rangle_{J}$ é o valor esperado do vácuo na presença de uma fonte externa $J(x)$. Quando $J(x) \rightarrow 0, \phi_{c l}(x)$ torna-se uma constante, $\phi_{c}$.

Agora, $m$ é um operador real, elíptico e auto-adjunto (por causa da continuação analítica), e para estes tipos de operadores nós podemos definir a assim chamada função zeta generalizada. Seja $\left\{\lambda_{i}\right\}$ os autovalores do operador $m(x, y)$. A função zeta generalizada associada ao operador $M(x, y)\left(m \rightarrow M=\frac{m}{2 \pi \mu^{2}}\right)$ é definida por

$$
\zeta_{M}(s)=\frac{1}{2} \frac{\hbar}{\Omega} \sum_{i}\left(\frac{\lambda_{i}}{2 \pi \mu^{2}}\right)^{-s}
$$

onde nós introduzimos um parâmetro de escala desconhecido $\mu$, com dimensões de inverso do comprimento $\left([L]^{-1}\right)$ ou massa $([M])$ para manter a função zeta adimensional para todo $s$. A introdução do parâmetro de escala $\mu$, pode ser mais bem compreendido quando nós observamos que existe escondida uma divisão da integral divergente no procedimento de regularização da função zeta, isto é, uma separação das partes divergente e finita do $V_{e f}\left(\phi_{c}\right)$ (em [4], pág. 208 e em [5], pág. 88). É bem conhecida a relação [2]

$$
\ln \operatorname{det} M=-\frac{d \zeta_{M}(0)}{d s}
$$

Agora, o potencial efetivo para a primeira ordem na expansão em "loop" pode ser escrito como

$$
V_{e f}^{(1)}\left(\phi_{c}\right)=-\frac{d \zeta_{M}(0)}{d s}
$$

O cálculo do potencial efetivo, usando-se a eq.(6), conduz a um resultado finito sem a necessidade de subtração de qualquer pólo ou a adição de contratermos infinitos. Isso é devido ao fato de que a função zeta generalizada como definida na eq.(4) é regular em $s=0$ $[2,8]$. Evidentemente, a necessidade de ajustar os parâmetros da teoria aos resultados observados nos leva a impor condições de renormalização, tais como

$$
\begin{aligned}
& \left.\frac{d^{2} V_{e f}}{d \phi_{c}^{2}}\right|_{\langle\phi\rangle}=m_{R}^{2}, \\
& \left.\frac{d^{4} V_{e f}}{d \phi_{c}^{4}}\right|_{\langle\phi\rangle}=\lambda_{R},
\end{aligned}
$$

onde $m_{R}$ é a massa renormalizada, $\lambda_{R}$ é a constante de acoplamento renormalizada e $\langle\phi\rangle$ é o ponto de mínimo do potencial efetivo (ponto de subtração ou renormalização) $[6,9]$.

No caso de teorias de massa nula, o ponto de subtração para a condição de renormalização (8) não pode ser tomado em $\langle\phi\rangle=0$ devido à singularidade logarítmica. Porém, nos casos de teorias de massa nula não existe uma escala intrínseca de massa, portanto todos os pontos de renormalização são equivalentes e a condição (8) é trocada por

$$
\left.\frac{d^{4} V_{e f}}{d \phi_{c}^{4}}\right|_{\langle\phi\rangle=M}=\lambda_{R}
$$

onde $M$ é um ponto de renormalização flutuante arbitrário [4].

Alternativamente, nós podemos usar a relação

$$
\ln \operatorname{det}[m(x, y)]=\operatorname{tr} \ln [m(x, y)],
$$

e obtermos [4]

$$
V_{e f}^{(1)}\left(\phi_{c}\right)=\frac{\hbar}{2} \int \frac{d^{4} k}{(2 \pi)^{4}} \ln \left[k_{E}^{2}+V^{\prime \prime}\left(\phi_{c}\right)\right],
$$

onde $k_{E}$ é o quadrimomento e o subíndice $\mathrm{E}$ denota espaço-tempo euclidiano. Como pode ser visto, a integral da eq.(11) é claramente divergente e, portanto, nós necessitamos um procedimento de regularização para isolar as divergências. Disto nós concluímos que a determinação do potencial efetivo, usando-se a função zeta, eq.(6), deve esconder o cancelamento das divergências de algum modo.

Com o propósito de mostrarmos explicitamente como o cancelamento das divergências ocorre, nós escrevemos os autovalores do operador $m(x, y)$ como

$$
\lambda_{i, \omega}=\omega^{2}+h_{i}^{2},
$$

onde $h_{i}$ são os autovalores do operador hamiltoniano $H$ e $\omega$ é um parâmetro contínuo designando a parte temporal dos autovalores do operador $m(x, y)$.

A função zeta associada ao operador $M(x, y)$, definida pela eq.(4), pode ser escrita, usando-se a eq.(12), como

$$
\zeta_{M}(s)=\frac{1}{2} \frac{\hbar}{\Omega} \int_{-\infty}^{\infty} \frac{d \omega}{2 \pi} \sum_{i}\left[\frac{\omega^{2}}{2 \pi \mu^{2}}+\frac{h_{i}^{2}}{2 \pi \mu^{2}}\right]^{-s} T .
$$

Usando a relação [10]

$\int_{-\infty}^{\infty}\left(k^{2}+A^{2}\right)^{-s} d^{m} k=\frac{\pi^{\frac{m}{2}} \Gamma(s-m / 2)}{\Gamma(s)}\left(A^{2}\right)^{\frac{m}{2}-s}$

nós podemos realizar a integral acima em $d \omega$ e obtermos

$$
\zeta_{M}(s)=\frac{1}{2 \sqrt{\pi}} \frac{\Gamma[s-1 / 2]}{\Gamma[s]} \frac{1}{2} \frac{\hbar}{\Omega} \zeta_{H}(s-1 / 2),
$$

onde $\zeta_{H}(s-1 / 2)$ é a função zeta generalizada associada ao operador hamiltoniano $H$, definida por

$$
\zeta_{H}(s-1 / 2)=\sqrt{2 \pi \mu^{2}} \sum_{i}\left(\frac{h_{i}^{2}}{2 \pi \mu^{2}}\right)^{1 / 2-s} .
$$


É bem conhecido que $\zeta_{M}(s)$ é analítica em $s=0[8$, $11,12]$. Portanto, $\zeta_{M}(0)$ é finita. Assim, ou $\zeta_{H}(s-1 / 2)$ é analítica em $s=0$ e $\zeta_{M}(0)$ é zero ou $\zeta_{H}(s-1 / 2)$ não é analítica em $s=0$ e neste caso $\zeta_{H}(s-1 / 2)$ deve ter a mesma estrutura de pólos simples da função gama $\Gamma(s)$, tal que $\zeta_{M}(s)$ seja analítica em $s=0$. Assim, a função zeta generalizada associada ao operador $H$ pode ser escrita como

$$
\zeta_{H}(s-1 / 2)=F(s)+D(s) \Gamma(s),
$$

onde $F(s)$ e $D(s)$ são funções analíticas em $s=0$. Notese que $s$ é o regulador usado no procedimento de regularização analítica da soma da energia de ponto-zero.

A eq.(17) é evidente quando nós realizamos a expansão em série de Laurent

$$
\begin{gathered}
\zeta_{H}(s-1 / 2)=\frac{a_{-1}}{s}+a_{0}+a_{1} s+a_{2} s^{2}+\ldots, \\
\zeta_{H}(s-1 / 2)=\frac{a_{-1} 2 \sqrt{\pi}}{\Gamma(s-1 / 2)} \Gamma(s)+a_{0}+a_{1} s+a_{2} s^{2}+\ldots,
\end{gathered}
$$

onde $D(s)$ e $F(s)$ são auto-evidentes. Uma vez que $\zeta_{H}(s-1 / 2)$ tem somente um pólo simples, a expansão é univocamente determinada.

Agora, diferenciando-se a eq.(15) e usando-se a eq.(17), nós obtemos

$$
\begin{aligned}
\zeta_{M}^{\prime}(s)= & \frac{1}{2} \frac{\hbar}{\Omega} \frac{\Gamma(s-1 / 2)}{2 \sqrt{\pi} \Gamma(s)}[\psi(s-1 / 2) F(s)+\psi(s-1 / 2) D(s) \Gamma(s)-\psi(s) F(s)+ \\
& \left.-\psi(\mathbf{s}) \boldsymbol{\Gamma}(\mathbf{s}) \mathbf{D}(\mathbf{s})+F^{\prime}(s)+D^{\prime}(s) \Gamma(s)+\mathbf{D}(\mathbf{s}) \psi(\mathbf{s}) \boldsymbol{\Gamma}(\mathbf{s})\right] .
\end{aligned}
$$

Observe-se que os termos em negrito são divergentes e cancelam-se mutualmente. Finalmente nós encontramos

$$
\zeta_{M}^{\prime}(0)=-\frac{1}{2} \frac{\hbar}{\Omega}\left[F(0)+\psi(-1 / 2) D(0)+D^{\prime}(0)\right] .
$$

$\mathrm{O}$ resultado acima explicitamente mostra como as divergências são canceladas na determinação do potencial efetivo usando-se a eq.(6). Portanto, é claro que escondido em $\zeta_{M}^{\prime}(0)$ existe um procedimento para obter a parte finita da eq.(11).

\section{Equivalência}

Uma vez que a eq.(6) fornece um resultado finito, deve existir, é claro, algum procedimento pelo qual se obtém, sem ambigüidades, a parte finita da teoria. Este procedimento foi empregado por Salam-Strathdee [13] e provado por Bollini-Giambiagi ser solução matemática para o problema [14]. O procedimento é muito simples: se $\Psi(s)$ é uma função com somente um pólo simples em, digamos, $s=0$, então sua parte finita é definida como

$$
F^{R}=\lim _{s \rightarrow 0}\left[\frac{\partial}{\partial s}(s \Psi(s))\right] .
$$

É importante notar que a eq.(21) tem fornecido a continuação analítica necessária para restabelecer a teoria original.

Agora, nós sabemos que a função zeta $\zeta_{M}(s)$ é analítica em $s=0$. Portanto, nós podemos escrevêla como

$$
\zeta_{M}(s)=-s f(s)
$$

onde a função $f(s)$ tem um pólo simples em $s=0$ para que a eq.(21) seja satisfeita.

O mínimo do potencial efetivo é a densidade de energia do vácuo que pode ser identificado à energia de ponto-zero (ZPE) [15]. A energia de ponto-zero é definida como

$$
\epsilon=\frac{\hbar T}{2 \Omega} \sum_{i} h_{i}
$$

onde $h_{i}$ são os autovalores do operador hamiltoniano os quais são dados por

$$
h_{i}^{2}=p^{2}+V_{c l}^{\prime \prime}(\bar{\phi}) .
$$


Assim,

$$
\epsilon=\frac{\hbar T}{2 \Omega} \sum \int d^{3} p \sqrt{p^{2}+V_{c l}^{\prime \prime}(\bar{\phi})}
$$

Naturalmente, o somatório (integral) acima é claramente divergente.

A fim de regularizarmos a expressão acima, vamos usar o conceito de continuação analítica ${ }^{1}$. A idéia é trocarmos a expressão original com um somatório (integral) divergente por uma função analítica bem comportada de um parâmetro complexo. O somatório (integral) agora é convergente e pode ser realizada formalmente sem ambigüidades. Após isso, pode-se, então, continuar analiticamente a expressão resultante para um valor do parâmetro complexo que restabeleça a teoria original. A divergência ultravioleta original reaparece como pólo neste valor do parâmetro complexo. A subtração deste pólo, por fim, conduz a um resultado finito [16]

Desta forma, vamos trocar $\epsilon$ por sua continuação analítica dada por

$$
\epsilon=\frac{\hbar T}{2 \Omega} \zeta_{H}(s-1 / 2)
$$

Em um procedimento de regularização analítica , como já dissemos, a divergência aparece como pólo simples no valor físico do parâmetro de regularização, s. Portanto, $\epsilon(s)$ é uma função meromórfica com um pólo simples em $s=0$ e, então, nós podemos escrevê-la como

$$
\epsilon(s)=\frac{b_{-1}}{s}+\sum_{n=0}^{\infty} b_{n} s^{n} .
$$

Para restabelecer a teoria original, livre de divergências, nós usamos o procedimento da eq.(21) na eq.(27). Nós obtemos a energia de ponto-zero renormalizada

$$
\epsilon^{R}=b_{0} \text {. }
$$

Uma vez que $\epsilon^{R}$ é a densidade de energia do vácuo, segue das eqs. (6) e (22) que

$$
f(s)=\epsilon(s) .
$$

A eq.(29) pode ser facilmente provada usando-se a eq.(15) e a identidade

$$
\frac{2 \sqrt{\pi} \Gamma[s]}{\Gamma[s-1 / 2]}=-\frac{1}{s},
$$

para obtermos

$$
\zeta_{M}(s)=-s \epsilon(s) .
$$

Comparando-se a eq.(22) e a eq.(31), nós encontramos o resultado da eq.(29). Agora, usando-se a eq.(31), nós podemos escrever

$$
\epsilon(s)=-\frac{1}{s} \zeta_{M}(s)
$$

Realizando um expansão em série de Laurent de $\epsilon(s)$

$$
\epsilon(s)=-\zeta_{M}(0) \frac{1}{s}-\zeta_{M}^{\prime}(0)-\zeta_{M}^{\prime \prime}(0) \frac{s}{2}-\ldots
$$

e usando a eq.(21), nós obtemos

$$
\epsilon^{R}=-\zeta_{M}^{\prime}(0)
$$

Portanto, quando nós usamos o método da função zeta, nós estamos, implicitamente, realizando um procedimento de regularização analítica, cuja parte finita é obtida como

$$
\lim _{s \rightarrow 0}\left[\frac{\partial}{\partial s}(s \epsilon(s))\right] .
$$

Naturalmente, a multiplicação de $\epsilon(s)$ por $s$ cancela o pólo evitando-se as possíveis divergências. Assim, nós afirmamos que o método da função zeta é completamente equivalente ao procedimento de regularização analítica para obtermos a energia do vácuo ( ou energia de ponto-zero). Nós não estamos afirmando aqui a equivalência entre o potencial efetivo e a energia de ponto-zero, uma vez que esta é uma afirmação mais profunda e já bem conhecida [16 - 18].

\section{Conclusão}

O procedimento de regularização do mínimo do potencial efetivo usando-se o método da função zeta nos fornece um resultado finito sem a necessidade de subtração de qualquer pólo ou a adição de contratermos infinitos.

Isso é devido ao fato de que a função zeta é regular em $s=0$. Desta maneira procedimentos dúbios usados para obter-se um resultado finito para o mínimo do potencial efetivo são evitados, uma vez que, como nós mostramos, as divergências são implicitamente canceladas pelo método da função zeta.

O método da função zeta para a determinação do potencial efetivo é implicitamente equivalente a um procedimento de regularização analítica para obter-se a energia de ponto-zero, cuja parte finita é obtida da eq.(21). Além disso, a eq.(21) fornece a continuação analítica necessária para restabelecermos a teoria original no caso da regularização analítica da energia de ponto-zero.

\section{Agradecimentos}

Nós gostariamos de agradecer à Agência Nacional para Pesquisa $(\mathrm{CNPq})($ Brasil) pelo suporte parcial deste trabalho.

\footnotetext{
${ }^{1}$ Seja $F_{1}$ uma função analítica em um domínio $D_{1}$ e $F_{2}$ uma função analítica em um domínio $D_{2}$. Se os domínios $D_{1}$ e $D_{2}$ se interceptam e em cada ponto da intersecção $D_{1} D_{2}$ as funções $F_{1}$ e $F_{2}$ são iguais, dizemos que $F_{2}$ é continuação analítica de $F_{1}$. É importante ressaltar o fato que a continuação $F_{2}$ de $F_{1}$ é única.
} 


\section{Referências}

[1] L.H. Ryder, Quantum Field Theory, Cambridge University Press, Cambridge (1985).

[2] P. Ramond, FIELD THEORY A Modern Primer, The Benjamin/Cummings Publishing Company, Inc., Massachusetts (1981).

[3] R.J. Rivers, Path Integral Methods in Quantum Field Theory, Cambridge University Press, Cambridge (1987).

[4] K. Huang, Quarks Leptons \& Gauge Fields, Word Scientific Publishing Company, Singapore (1982).

[5] J.V. Narlikar and T. Padmanabhan, Gravity, Gauge Theories and Quantum Cosmology, D. Reidel Publishing Company, Dordrecht (1986).

[6] J. Iliopoulos, C. Itzykson and A. Martin, Rev. Mod. Phys., 47, (1975) 165.

[7] R. Jackiw, Phys. Rev. D 9(6), (1974) 1686.

[8] S. Hawking, Commun. Math. Phys. 55, (1977) 133.

[9] S. Coleman and E. Weinberg, Phys. Rev. D7(6), (1973) 1888.
[10] E. Myers, Phys. Rev. Lett. 54, (1987) 165.

[11] N. D. Birrell and P. C. W. Davies, Quantum Fields in Curved Space, Cambridge University Press, Cambridge, (1982).

[12] S. K. Blau, M. Visser and A. Wipf, Nucl. Phys. B 310, (1988) 163.

[13] A. Salam and J. Strathdee, Nucl. Phys. B90, (1975) 203.

[14] C. G. Bollini and J. J. Giambiagi, Il Nuovo Cimento 31(3), (1964) 550

[15] T. H. Boyer, Ann. Phys. 56, (1970) 474.

[16] G. Leibbrandt, Rev. Mod. Phys. 47(4), (1975) 849.

[17] T. Treml, Can. J. Phys. 68, (1990) 91.

[18] J. A. Nogueira and A. Maia Jr., Phys. Lett. B358, (1995) 56.

[19] J. A. Nogueira and A. Maia Jr., Phys. Lett. B394, (1997) 371 